Orignal Article

\title{
Predictive factors for response and toxicity after brachytherapy for rectal cancer; results from the HERBERT study
}

\author{
Eva C. Rijkmans ${ }^{\mathrm{a}, *}$, Corrie A.M. Marijnen ${ }^{\mathrm{a}}$, Baukelien van Triest ${ }^{\mathrm{b}}$, Martijn Ketelaars ${ }^{\mathrm{a}}$, Annemieke Cats ${ }^{\mathrm{c}}$, \\ Akin Inderson $^{d}$, Roy P.J. van den Ende ${ }^{a}$, Mirjam S. Laman ${ }^{a}$, Ellen M. Kerkhof ${ }^{a}$, Remi A. Nout ${ }^{a}$

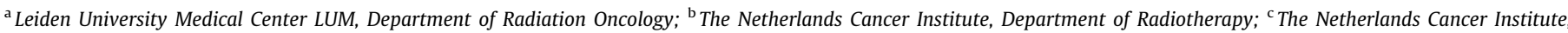 \\ Department of Gastrointestinal Oncology, Amsterdam; and ${ }^{\mathrm{d}}$ Leiden University Medical Center LUMC, Department of Gastroenterology and Hepatology Leiden, the Netherlands
}

\section{A R T I C L E I N F O}

\section{Article history:}

Received 10 October 2018

Received in revised form 24 January 2019

Accepted 27 January 2019

Available online 18 February 2019

Presented at the 36th ESTRO conference,

05-09 May, 2017, Vienna, Austria and the

37th ESTRO conference, 20-24 April, 2018,

Barcelona, Spain.

\section{Keywords:}

Rectal cancer

Brachytherapy

DVH

Dose-response

Clinical complete response

Toxicity

\begin{abstract}
A B S T R A C T
Purpose: The HERBERT study was a dose-finding feasibility study of a high-dose rate endorectal brachytherapy (HDREBT) boost after external beam radiotherapy (EBRT) in elderly patients with rectal cancer who were unfit for surgery. This analysis evaluates the association of patient, tumor and dosimetric parameters with tumor response and toxicity after HDREBT in definitive radiotherapy for rectal cancer.

Patients and methods: The HERBERT study included 38 inoperable patients with T2-3N0-1 rectal cancer. Thirteen fractions of 3 Gy EBRT were followed by three weekly HDREBT applications of 5-8 Gy per fraction. Clinical and dosimetric parameters were tested for correlation with clinical complete response (cCR), sustained partial/complete response (SR), patient reported bowel symptoms, physician reported acute and late proctitis (CTCAE v3) and endoscopically scored toxicity.

Results: Thirty-five patients completed treatment and were included in the current analyses. Twenty of 33 evaluable patients achieved a CCR, the median duration of a sustained response was 32 months. Tumor volume at diagnosis showed a strong association with clinical complete response (OR $1.15 ; p=0.005$ ). No dose-response correlation was observed in this cohort. Prescribed dose to the brachytherapy CTV (D90) correlated with acute and late physician reported proctitis while CTV volume, CTV width and high dose regions in the CTV (D1cc/D2cc) were associated with endoscopic toxicity at the tumor site.

Conclusion: Tumor volume is the most important predictive factor for tumor response and a higher dose to the brachytherapy CTV increases the risk of severe clinically and endoscopically observed proctitis after definitive radiotherapy in elderly patients with rectal cancer.
\end{abstract}

(c) 2019 Elsevier B.V. All rights reserved. Radiotherapy and Oncology 133 (2019) 176-182
Over the last decades, radiotherapy for rectal cancer has developed substantially. While total mesorectal excision (TME) with or without neo-adjuvant (chemo)radiotherapy remains the gold standard, risk of surgical morbidity and mortality and the possibility of a clinical or pathologic complete response after neo-adjuvant treatment have led to increasing interest in organ preservation strategies $[1,2]$. Especially in elderly fragile patients with multiple comorbidities, surgical risks might outweigh the possible improved long-term oncological outcome [3]. With rising awareness for organ preservation, it is important to understand which factors are associated with a complete response. Previous studies describe a radiotherapy dose-effect relationship for rectal cancer $[4,5]$. A possible option for dose escalation is the use of

* Corresponding author at: Albinusdreef 2, 2300RC Leiden, PO Box 9600, Zone K1$P$, the Netherlands.

E-mail address: e.c.rijkmans@lumc.nl (E.C. Rijkmans). high-dose rate endorectal brachytherapy (HDREBT), which allows for high doses to the tumor with sparing of the surrounding organs.

The HERBERT study was a dose escalation study, performed to evaluate the feasibility of a HDREBT boost after external beam radiotherapy (EBRT) in elderly patients with T2-3N0-2 rectal cancer who were unfit for standard TME surgery. The primary endpoint was the maximum tolerated brachytherapy boost dose after 13x3Gy external beam radiotherapy. This was set at $3 \times 7 \mathrm{~Gy}$ after three patients in the dose level with $8 \mathrm{~Gy}$ per fraction experienced acute grade 3 proctitis [6].

Of the 38 patients included in the study, 35 completed brachytherapy treatment and 33 had endoscopic follow-up and were therefore evaluable for response analyses. Overall response was high (90\% complete or partial response) and $60 \%$ achieved a clinical complete response. Severe toxicity was however not uncommon and occurred in approximately one third of patients [6]. 
Few other studies have used HDREBT in treatment of rectal cancer. Treatment schedules between these studies vary widely and no consensus exists on dose prescription, fractionation or constraints for organs at risk [7]. Therefore it is of importance to better understand which patient, tumor and treatment characteristics have predictive value for tumor response and toxicity. This will aid in selection of patients who are most likely to benefit from a HDREBT boost. The aim of the current analyses is to evaluate patient, tumor and treatment parameters in relation to tumor response and toxicity in the HERBERT study.

\section{Materials and methods}

A dose finding feasibility study was performed from 2007 to 2013 in elderly or medically inoperable patients with cT2-3N0-2 rectal cancer. Patients received 13 fractions of 3 Gy EBRT followed by three weekly HDREBT applications of 5, 6, 7 or $8 \mathrm{~Gy}$ per fraction six weeks after EBRT. Details of the study design and methods have been described previously $[6,8]$.

\section{Endorectal brachytherapy}

For HDREBT a flexible applicator with a central canal and 8 peripheral catheters (Intracavitary Mold Applicator, ELEKTA, Veenendaal, The Netherlands) was used in combination with an inflatable semi-circular balloon to fixate the applicator and push away the normal rectal wall. HDREBT was performed with an Iridium192 source using a microSelectron HDR after loader (Elekta, Veenendaal, the Netherlands). Treatment planning was performed with Oncentra Brachy (Elekta, Veenendaal, the Netherlands) on a planning-CT with applicator in situ acquired prior to the first brachytherapy application. The aim of treatment planning was complete coverage of the clinical target volume (CTV) by the $100 \%$ isodose with no hotspots in the surrounding organs. The CTV was defined as the area suspicious for residual tumor and/or scarring at time of brachytherapy. Delineation was performed on the planning-CT by two observers using information of the diagnostic MRI, endoluminal clips at the proximal and distal border of the tumor, and rectoscopy images with a clinical drawing acquired prior to EBRT and during the first brachytherapy session. In case of discrepancy between observers, consensus was sought for the definitive CTV $[6,9]$. The $100 \%$ isodose was restricted to $2 \mathrm{~cm}$ from the applicator surface and during the course of the study an additional constraint of $400 \%$ at the applicator surface was added. The brachytherapy treatment plan of the first fraction was used for the 2nd and 3rd fraction. Orthogonal x-rays, visualizing endoluminal clips that were placed at the borders of the tumor, allowed for position verification at time of each brachytherapy fraction [10].

\section{Tumor characteristics and DVH parameters}

The tumor was delineated on the diagnostic MRI to assess the baseline tumor volume. The HDREBT CTV, contralateral rectal wall and anal canal were delineated on the HDREBT planning-CT. The contralateral rectal wall was defined as the rectal wall, excluding lumen and CTV, extending $3 \mathrm{~cm}$ proximal and distal to the CTV. Fig. 1 shows two examples of contoured structures and HDREBT planning. Dose-volume histogram (DVH) parameters were collected from the initial planning-CT for CTV coverage: CTV D98 and CTV D90 and for high dose regions in the CTV; D1cc, D2cc and D5cc and dose in the contralateral wall and the anal canal: D2cc and D5cc. A point dose on the lumen side of the contralateral wall directly opposing the center of the CTV was also collected. All doses are described as physical dose per HDREBT fraction (Gy/\#). In addition, the isodose volumes, including applicator and lumen, corresponding to a cumulative total $2 \mathrm{~Gy}$ equivalent total dose (EQD2) of $60 \mathrm{~Gy}$ and $75 \mathrm{~Gy}$ were acquired using an $\alpha / \beta=3$ for late toxicity endpoints and $\alpha / \beta=10$ for tumor control [4] and acute toxicity.

\section{Endpoints}

Clinical tumor response evaluation was based on digital rectal examination and endoscopy and was performed prior to brachytherapy, at two and six months and yearly after brachytherapy. Maximum response was determined, including information of multiple endoscopic evaluations if needed, and was categorized as clinical complete response (cCR), partial response (PR; $>30 \%$ decrease of tumor bulk on endoscopy images), stable disease (SD; $\leq 30 \%$ decrease or $\leq 20 \%$ increase) or progressive disease (PD $>20 \%$ increase in tumor bulk on endoscopy images). Complete responders $(\mathrm{CCR})$ were compared to non-complete responders (no cCR). Sustained response was defined as complete or partial response with time calculated from start of EBRT to progression. Patients were censored at time of death or loss of follow-up.

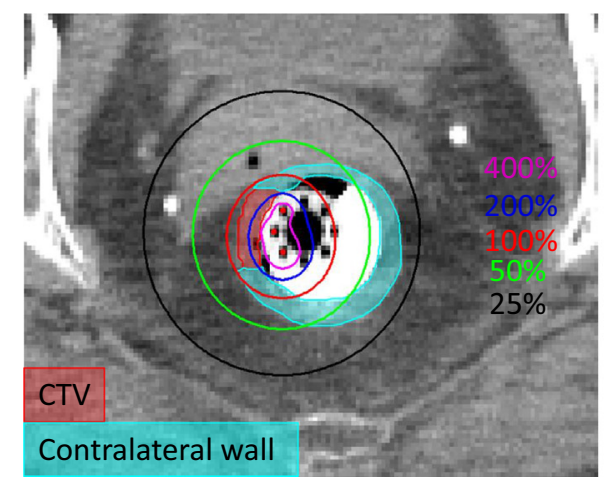

Doselevel 8Gy HDREBT CTV D98: $8.23 \mathrm{~Gy}$ HDREBT CTV D90: $9.22 \mathrm{~Gy}$ HDREBT CTV D2cc: $11.98 \mathrm{~Gy}$

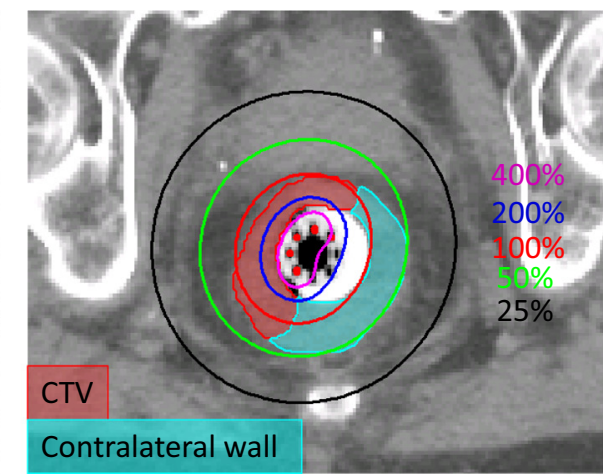

Dose level $8 \mathrm{~Gy}$ HDREBT CTV D98: $\quad 4.09 \mathrm{GY}$ HDREBT CTV D90: $5.08 \mathrm{GY}$ HDREBT CTV D2cc: $16.17 \mathrm{~Gy}$

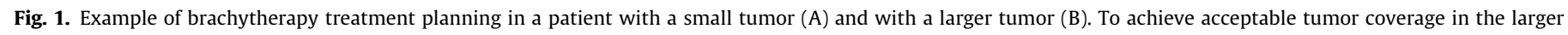
tumor, dose to the HDREBT CTV D2cc is increased. As a result, tumor volume and the CTV D2cc showed are correlated. 
Evaluation of clinical and endoscopic toxicity has been previously reported [9]. In summary, toxicity was assessed via three methods: (1) Patient reported bowel symptoms (PROM), assessed by questionnaires acquired from start of HDREBT to 2 months after HDREBT. Symptoms concerning pain with stools, painful abdominal cramps/urge, tenesmus, mucus discharge, fecal incontinence and bowel function in general were scaled, (2) Clinical toxicity (CTCAE v3): acute and late clinical proctitis and late $\geq$ grade 2 rectal bleeding and incontinence. (3) Endoscopic evaluation: endoscopically scored toxicity at the tumor site was categorized as 0 . erythema or scarring, 1 . superficial ulcer and 2 . (very) deep ulcer, and toxicity at the contralateral wall as 0 . normal mucosa; 1 . mild erythema; 2. diffuse erythema and punctate hemorrhage; 3 . frank hemorrhage and 4. ulceration [11]. Patients with SD or PD were excluded for late toxicity and evaluation of endoscopically scored toxicity at the tumor site.

\section{Statistical analyses}

Statistical analyses were performed with SPSS version 23.0 (IBM, Armonk, NY). Correlation of factors was assessed using Spearman's correlation. Further analyses for association were performed with the Armitage test for trend for ordinal variables and the Kruskal-Wallis $\mathrm{H}$ and Mann-Whitney $U$ test for continuous variables. Logistic regression and cox-regression were used for uni- and multivariable analyses. Due to small sample size, multivariable analyses were only performed for binary outcome measures with a maximum of 2 variables. A $p$-value $<0.01$ was considered significant to correct for multiple testing.

\section{Results}

Thirty-five of 38 patients included in the study completed treatment and were included in the current analyses. All 35 patients were available for evaluation of acute toxicity, 26 for late toxicity and 33 for response evaluation. Baseline patient characteristics have been previously reported [6]. Patients were mainly elderly with a median age of 83 years and most patients had severe comorbidity, with $80 \%$ classified as American Society of Anesthesiology III to IV and $69 \%$ anticoagulant use. Twenty patients had a cT2 tumor and fifteen a cT3 tumor. Of these fifteen, five had a tumor with $>5 \mathrm{~mm}$ fat infiltration (cT3c/d). MRI showed positive nodes in 11 patients; N1 in 9 patients and N2 in 2 patients. Baseline tumor characteristics and brachytherapy dose-volume histogram (DVH) parameters are listed in Table 1.

\section{Clinical tumor response}

After full treatment, clinical complete response was achieved in 20 of 33 evaluable patients. Seven of these patients already had a cCR after EBRT alone. A sustained partial or complete response (SR) was seen in $61.8 \%$ at one year, $54.7 \%$ at 2 years and $46.5 \%$ at three years, with a median SR of 32 months. Table 2 shows the results of the univariable analyses for CCR and for SR. Volume at baseline was the only significant predictive factor for clinical complete response; OR 1.15 (cc) $p=0.005$. Median volume of patients with a complete response was $10.8 \mathrm{cc}$ vs. $27.3 \mathrm{cc}$ in patients without cCR (see Fig. 2A). Clinical nodal stage, response to EBRT, tumor thickness at baseline and HDREBT CTV circumference all showed a trend for association with cCR. In patients with positive lymph nodes, complete tumor response rate was $36 \%$ compared to $73 \%$ in cNO patients $(p=0.05)$. Thirteen of twenty-one patients with a partial response after EBRT achieved a CCR after HDREBT, while none of five patients with stable disease achieved a cCR $(p=0.01)$. For sustained response a trend was observed for tumor
Table 1

Tumor and treatment parameters.

\begin{tabular}{|c|c|c|}
\hline & $n$ & $\%$ \\
\hline \multicolumn{3}{|l|}{ Clinical tumor stage } \\
\hline cT2 & 20 & $57.1 \%$ \\
\hline сT3 & 15 & $42.9 \%$ \\
\hline cN1-2 & 11 & $31.4 \%$ \\
\hline Baseline tumor measurements & Median & Range \\
\hline Tumor volume $(\mathrm{cc})^{*}$ & 11.3 & $2.1-39.8$ \\
\hline Tumor thickness (mm) & 16 & $5-34$ \\
\hline Tumor length $(\mathrm{cm})^{\dagger}$ & 4.0 & $1.5-6.0$ \\
\hline Distance from anal verge $(\mathrm{cm})^{\dagger}$ & 6.0 & $2.0-15.0$ \\
\hline Tumor circumference $(\%)^{\dagger}$ & 40 & $15-90$ \\
\hline \multicolumn{3}{|l|}{ HDREBT CTV characteristics } \\
\hline CTV volume $(\mathrm{cc})^{*}$ & 7.2 & $2.0-25.0$ \\
\hline CTV max thickness $(\mathrm{mm})$ & 10 & $4-30$ \\
\hline CTV length $(\mathrm{cm})^{*}$ & 3.1 & $1.8-6.4$ \\
\hline CTV width $(\mathrm{cm})$ & 3.8 & $1.0-7.9$ \\
\hline CTV circumference $\%$ & 30 & $20-80$ \\
\hline \multicolumn{3}{|l|}{ HDREBT DVH parameters per fraction } \\
\hline CTV D98 (Gy/\#) & 6.0 & $1.2-8.8$ \\
\hline CTV D90 (Gy/\#) & 7.2 & $1.8-9.8$ \\
\hline CTV D1cc (Gy/\#) & 14.9 & $7.9-28.4$ \\
\hline CTV D2cc (Gy/\#) & 12.9 & $5.3-22.5$ \\
\hline CTV D5cc (Gy/\#) & 9.2 & $3.6-15.0$ \\
\hline \multicolumn{3}{|l|}{ Contralateral rectal wall } \\
\hline Contralateral wall D2cc (Gy/\#) & 8.1 & $3.7-14.2$ \\
\hline Contralateral wall D5cc (Gy/\#) & 5.9 & $2.6-11.6$ \\
\hline Point dose contralateral wall (Gy/\#) & 5.0 & $1.7-18.7$ \\
\hline \multicolumn{3}{|l|}{ Anal canal } \\
\hline Anal canal D2cc (Gy/\#) & 1.2 & $0.0-4.4$ \\
\hline Anal canal D5cc (Gy/\#) & 1.0 & $0.0-3.2$ \\
\hline \multicolumn{3}{|l|}{ Volume cumulative dose ${ }^{\S}$} \\
\hline Volume EQD2 $60 \mathrm{~Gy} \alpha / \beta=3$ (cc) & 102.9 & $26.2-203.1$ \\
\hline Volume EQD2 $60 \mathrm{~Gy} \alpha / \beta=10$ (cc) & 61.9 & $16.9-123.6$ \\
\hline Volume EQD2 $75 \mathrm{~Gy} \alpha / \beta=3$ (cc) & 50.0 & $13.7-104.4$ \\
\hline Volume EQD2 $75 \mathrm{~Gy} \alpha / \beta=10$ (cc) & 33.0 & $9.0-70.3$ \\
\hline
\end{tabular}

Abbreviations: HDREBT, High dose rate endorectal brachytherapy. EQD2, equivalent dose in 2 Gy fractions. Gy/\#, Gy per brachytherapy fraction.

Based on delineation on diagnostic MRI.

Based on diagnostic endoscopy and MRI.

* Based on HDREBT planning-CT.

$\S$ Volume derived from isodose lines (including applicator) corresponding to a cumulative dose of 60 and $75 \mathrm{~Gy}$.

volume, circumferential involvement and thickness at baseline and for HDREBT CTV volume, length and circumferential involvement (see Table 2). The effect of tumor volume at time of diagnosis and at time of brachytherapy on complete and sustained response is illustrated in Fig. 2. Patients with a baseline tumor volume $<20 \mathrm{cc}$ had a 2 year sustained response rate of $74 \%$ compared to only $25 \%$ for patients with baseline tumor volume $>20 \mathrm{cc}(p=0.007)$.

No dose-response correlation was observed for HDREBT CTV dose coverage (D98/D90). After correction for CTV volume in multivariable analyses still no association could be established. In univariable analyses HDREBT CTV high dose regions (D1cc/D2cc) showed a negative association with CCR and SR. CTV D2cc was however correlated to CTV volume ( $\rho=0.63 ; p<0.001$ ), illustrated in Fig. 1) and after correction for CTV volume in multivariable analyses this association was no longer detected.

\section{Toxicity}

Clinically relevant correlations between patient, tumor and DVH parameters with toxicity endpoints are shown in Table 3. Full analyses are available online in Web appendix A.

\section{Acute toxicity}

For patient reported bowel symptoms, a dose-response association was found for all DVH parameters of the HDREBT CTV, with 
Table 2

Univariable analyses for clinical complete response and local control.

\begin{tabular}{|c|c|c|c|c|c|c|}
\hline & \multicolumn{3}{|c|}{ Complete response } & \multicolumn{3}{|c|}{ Sustained response (CR/PR) } \\
\hline & OR & $(95 \% \mathrm{CI})$ & $p$ & HR & $(95 \% \mathrm{CI})$ & $p$ \\
\hline Baseline & & $n=33$ & & & $n=33$ & \\
\hline cT-stage (cT3 vs. cT2) & 1.75 & $(0.43-7.17)$ & 0.44 & 1.32 & $(0.50-3.53)$ & 0.58 \\
\hline cN-stage (N1-2 vs. N0) & 4.67 & $(0.99-21.9)$ & 0.05 & 2.15 & $(0.80-5.79)$ & 0.13 \\
\hline Volume at baseline $(\mathrm{cc})$ & 1.15 & $(1.04-1.27)$ & 0.005 & 1.05 & $(1.01-1.09)$ & 0.02 \\
\hline Circumference at baseline (per $10 \%$ ) & 1.49 & $(0.99-2.26)$ & 0.06 & 1.33 & $(1.04-1.68)$ & 0.02 \\
\hline Thickness at baseline (mm) & 1.16 & $(1.02-1.33)$ & 0.02 & 1.08 & $(1.01-1.16)$ & 0.03 \\
\hline Length at baseline $(\mathrm{cm})$ & 1.23 & $(0.72-2.10)$ & 0.45 & 1.06 & $(0.75-1.51)$ & 0.73 \\
\hline Distance to anal verge $(\mathrm{cm})$ & 0.99 & $(0.81-1.21)$ & 0.91 & 0.98 & $(0.85-1.13)$ & 0.78 \\
\hline HDREBT & & $n=26^{*}$ & & & $n=33$ & \\
\hline CTV volume $(\mathrm{cc})$ & 1.29 & $(0.98-1.69)$ & 0.07 & 1.11 & $(1.01-1.21)$ & 0.03 \\
\hline CTV circumference (per 10\%) & 2.21 & $(1.09-4.47)$ & 0.03 & 1.36 & $(1.04-1.78)$ & 0.03 \\
\hline CTV thickness (mm) & 1.23 & $(0.96-1.56)$ & 0.10 & 1.05 & $(0.96-1.15)$ & 0.31 \\
\hline CTV length $(\mathrm{cm})$ & 1.64 & $(0.57-4.73)$ & 0.36 & 1.64 & $(1.02-2.63)$ & 0.04 \\
\hline CTV width (cm) & 1.59 & $(0.76-3.34)$ & 0.22 & 1.34 & $(0.96-1.87)$ & 0.08 \\
\hline CTV D98 (Gy) & 1.02 & $(0.62-1.69)$ & 0.93 & 1.22 & $(0.82-1.80)$ & 0.33 \\
\hline CTV D90 (Gy) & 0.98 & $(0.62-1.55)$ & 0.93 & 1.20 & $(0.84-1.73)$ & 0.32 \\
\hline CTV D1cc (Gy) & 1.16 & $(0.93-1.43)$ & 0.19 & 1.13 & $(1.02-1.26)$ & 0.03 \\
\hline CTV D2cc (Gy) & 1.28 & $(0.94-1.74)$ & 0.12 & 1.19 & $(1.03-1.38)$ & 0.02 \\
\hline
\end{tabular}

HDREBT; high-dose rate endorectal brachytherapy, CTV; clinical target volume.

Significant results ( $p$ values $<0.01$ ) in bold and trends ( $p$ value $0.01-0.05$ ) in italics.

Effect of HDREBT variables on cCR were tested in patients with PR/SD at time of brachytherapy.
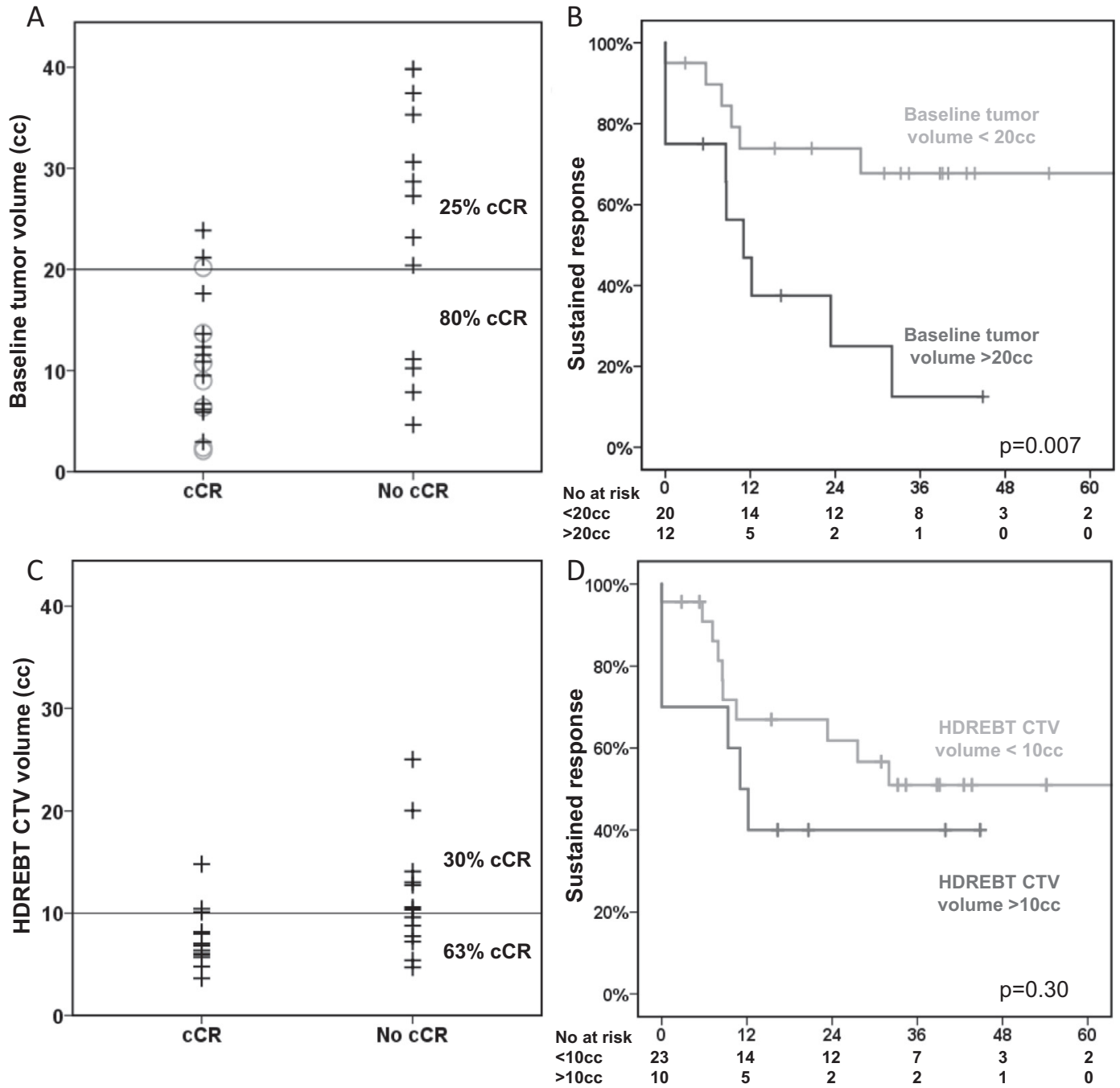

Complete response

Time from start of EBRT in months

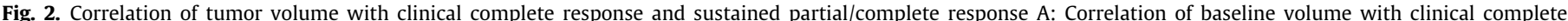

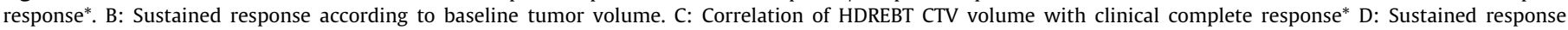
according to HDREBT CTV volume. *Patients with a cCR after EBRT are displayed in gray circles in Figure A and are excluded in Figure C. 
Table 3

Factors associated with toxicity.

\begin{tabular}{llll}
\hline & $\rho$ & $p$-Value & Explained variance \\
\hline PROM acute proctitis scale & & & \\
CTV D98 (Gy/\#) & 0.43 & 0.03 & 18.2 \\
CTV D90 (Gy/\#) & 0.46 & 0.02 & 20.7 \\
CTV D1cc (Gy/\#) & 0.56 & $\mathbf{0 . 0 0 3}$ & 31.4 \\
CTV D2cc (Gy/\#) & 0.51 & $\mathbf{0 . 0 0 7}$ & 25.9 \\
CTV D5cc (Gy/\#) & 0.50 & 0.03 & 24.5 \\
CTCAE acute proctitis & & & \\
Tumor thickness at baseline (mm) & 0.44 & $\mathbf{0 . 0 0 5}$ & 19.5 \\
CTV D98 (Gy/\#) & 0.43 & 0.01 & 18.4 \\
CTV D90 (Gy/\#) & 0.46 & $\mathbf{0 . 0 0 6}$ & 21.0 \\
CTCAE severe late proctitis & & & \\
CTV D90 (Gy/\#) & 0.43 & 0.03 & 18.5 \\
Endoscopic toxicity at the tumor site & & \\
CTV volume (cc) & 0.44 & 0.03 & 19.4 \\
CTV width (cm) & 0.53 & $\mathbf{0 . 0 0 6}$ & 28.1 \\
CTV D1cc (Gy/\#) & 0.42 & 0.03 & 21.0 \\
CTV D2cc (Gy/\#) & 0.59 & $\mathbf{0 . 0 0 1}$ & 41.7 \\
Volume EQD2 60 Gy (cc) & 0.55 & $\mathbf{0 . 0 0 4}$ & 26.2 \\
Volume EQD2 75 Gy (cc) & 0.57 & $\mathbf{0 . 0 0 3}$ & 26.6 \\
\hline
\end{tabular}

PROM; patient reported outcome measure, CTV; clinical target volume, Gy/\#; Gy per brachytherapy fraction.

Only factors with a trend or significant correlation are listed. Results of the complete analyses are available online.

Statistics: Spearman's correlation; significant results ( $p$ values $<0.01$ ) in bold and trends ( $p$ value $0.01-0.05$ ) in italics.

the strongest correlation of $\rho=0.56$ for CTV D1cc. Acute physician reported toxicity (CTCAE v3) was correlated to tumor thickness at baseline and HDREBT CTV D90 and D98. Median HDREBT CTV D90 was 6.6 Gy/\# (range 4.7-9.3 Gy) for grade $\leq 1$; $7.8 \mathrm{~Gy} / \#$ (range 1.8$9.8 \mathrm{~Gy}$ ) for grade 2 and $8.7 \mathrm{~Gy} / \#$ (range $7.2-9.8 \mathrm{~Gy}$ ) for grade 3 acute proctitis ( $p=0.04$; see Fig. $3 \mathrm{~A})$.

\section{Late proctitis}

Severe late proctitis ( $\geq$ grade 3 proctitis CTCAE v3) occurred in $10 / 25$ patients who achieved a CCR or CPR. Only HDREBT CTV D90 showed a correlation (see Table 3 and Fig. 3). Severe late proctitis occurred in $0 / 5(0 \%)$ patients with a CTV D90 <6 Gy/\#, in $5 / 13(38 \%)$ patients with a CTV D90 between 6 and $8 \mathrm{~Gy} / \#$ and in $5 / 7$ (71\%) patients with a CTV D90 exceeding $8 \mathrm{~Gy} / \#$ $(p=0.02)$. In multivariable analyses the effect of CTV D90 remained correlated to severe late proctitis after correction for tumor volume (CTV D90 OR 3.1 (Gy) $p=0.03$ and CTV volume OR $1.3 p=0.08$ ).

For late rectal bleeding, a trend was observed for use of anticoagulants: grade $\geq 2$ rectal bleeding occurred in $1 / 6$ patients without anticoagulants and in $12 / 19$ patients with anticoagulant use $(p=0.05)$. Late incontinence was not associated with any of the clinical or dosimetric parameters (data not shown).

\section{Endoscopic toxicity}

Endoscopic toxicity at the contralateral wall showed no correlation with clinical or dosimetric parameters (see Web appendix A). Endoscopic toxicity at the tumor site was correlated with HDREBT CTV volume and width, CTV D1cc and D2cc and volumes of 60 and $75 \mathrm{~Gy}\left(\mathrm{EQD} 2_{\alpha / \beta 3}\right)$, showing the strongest correlation of $\rho=0.59$ for CTV D2cc. Median CTV D2cc was $10.1 \mathrm{~Gy} / \#$ (5.3-12.9) in patients with erythema or scarring, $12.1 \mathrm{~Gy} / \#$ (7.7-17.0) in patients with a superficial ulcer and $14.8 \mathrm{~Gy} / \#$ (10.1-22.5) in patients with a deep or very deep ulcer (see Fig. 3D). Deep ulceration occurred in $7 / 9$ (78\%) of patients with a CTV D2cc $>14 \mathrm{~Gy} / \#$ and in 3/18 (17\%) patients with a CTV $\mathrm{D} 2 \mathrm{cc}<14 \mathrm{~Gy} / \#(p=0.002)$.

\section{Discussion}

This sub-analysis of the HERBERT study evaluated factors associated with tumor response and toxicity after a combination of EBRT and a brachytherapy boost in patients with rectal cancer. The results show that the most important predictor for a clinical complete response is tumor volume at baseline. Other factors that were associated with $\mathrm{CCR}$ included limited tumor thickness at baseline, a good response to EBRT and limited circumferential involvement at time of brachytherapy. Tumor volume, thickness and circumference at baseline were associated with a sustained partial/complete response. While we could not demonstrate a relation between brachytherapy dose and tumor response, a dose-effect relationship was observed for most toxicity endpoints. Prescribed dose to the brachytherapy CTV (D90) correlated best with acute and late physician reported proctitis and high dose regions in the CTV (D1cc/D2cc) were associated with patient reported toxicity and endoscopic toxicity at the tumor site.

The current analysis provides unique data on factors associated with response and toxicity after HDREBT for rectal cancer. While reports on HDREBT are limited, previous studies on definitive radiotherapy using contact-X-ray have demonstrated that $\mathrm{T}$ stage, tumor size, mobility/depth of invasion and early response to radiotherapy are strong prognostic factors for complete clinical tumor response [12-16]. Studies evaluating pathologic complete response after preoperative (chemo)radiotherapy have further shown a correlation with $\mathrm{cT}, \mathrm{cN}$ and $\mathrm{cM}$ stage, histological subtype (in favor of adenocarcinoma), differentiation grade, presence of vascular or lymphatic invasion (LVSI), the addition of neoadjuvant chemotherapy, radiotherapy dose escalation and timing from CRT to surgery [4,17-21].

Factors associated with tumor response in the current study were tumor volume, thickness, circumferential involvement, cNstage and response to EBRT. cT-stage showed no correlation, but it has long been recognized that tumor volume is a stronger predictor than cT-stage $[22,23]$. While there was substantial variation in total cumulative CTV D90, with a median EQD2 $2_{\alpha / \beta 10}$ of $72.9 \mathrm{~Gy}$ (IQrange: $68-80 \mathrm{~Gy}$ ) we did not observe a dose-response correlation. This can probably mainly be contributed to the limited number of 35 patients. The effect of dose escalation could also have been overshadowed by other factors such as tumor volume. Also, the use of one planning-CT scan for 3 fractions could have resulted in a different dose coverage at the 2nd and 3rd fraction limiting the dose-response analyses $[9,24]$. In addition, while aided by clips, MRI and endoscopy, soft tissue resolution on CT is poor and residual uncertainty in delineation may occur [25,26]. A negative correlation was observed for high dose regions to the brachytherapy CTV, which was interpreted, and confirmed by multivariable analysis, as an indirect effect of tumor volume.

Rectal morbidity after radiotherapy is a well-known problem and is especially challenging in definitive radiotherapy for rectal cancer given that the tumor is incorporated in the organ at risk. Only one study has prospectively evaluated toxicity after chemoradiation and a HDR boost. Cumulative EQD2 was 66 Gy and although overall functional outcome was good, rectal bleeding was present in approximately $80 \%>1$ year after treatment [27]. This study is the first to evaluate prognostic factors for radiation proctitis in patients with rectal cancer. From previous studies in patients with prostate and gynecological malignancies we know that radiation dose and co-morbidity, including diabetes mellitus and hemorrhoids, have been associated with increased risk of acute and late rectal toxicity [28]. Additional risk factors for late rectal morbidity include age, history of abdominal surgery, presence of cardiovascular disease, use of anticoagulants, smoking and the presence of acute rectal toxicity [28-33]. 
A

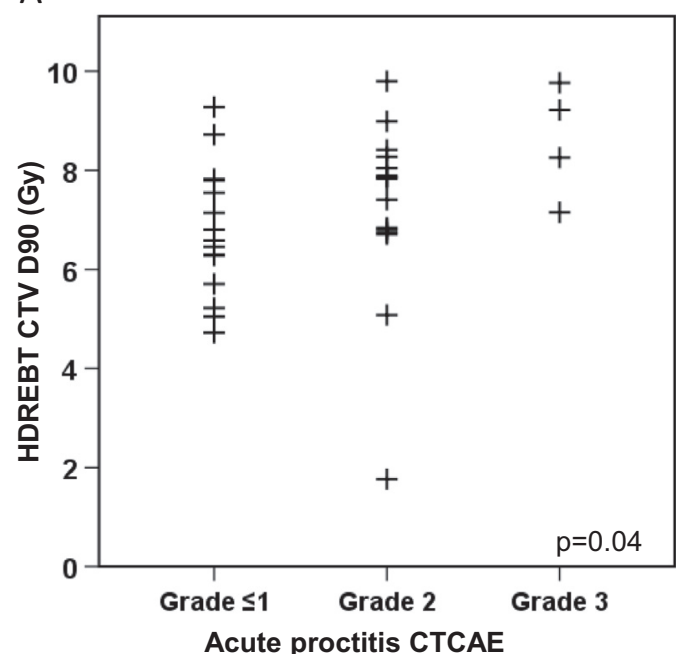

C

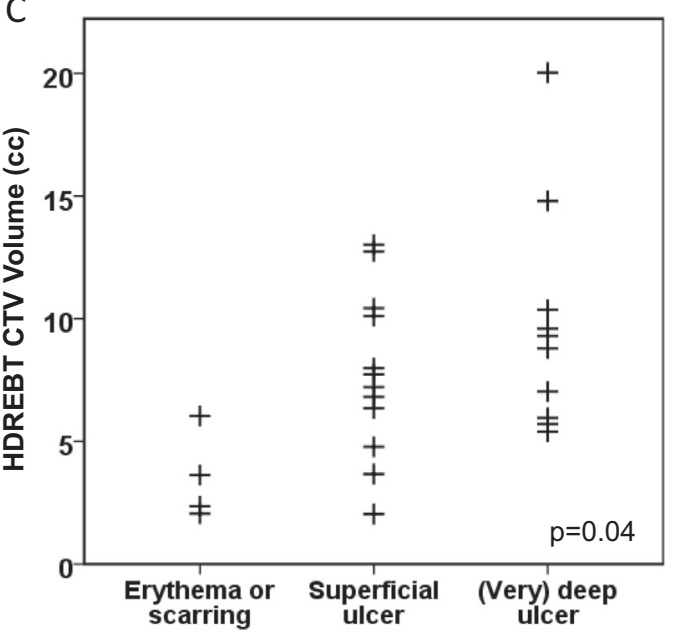

Endoscopic toxicity at tumor site
B

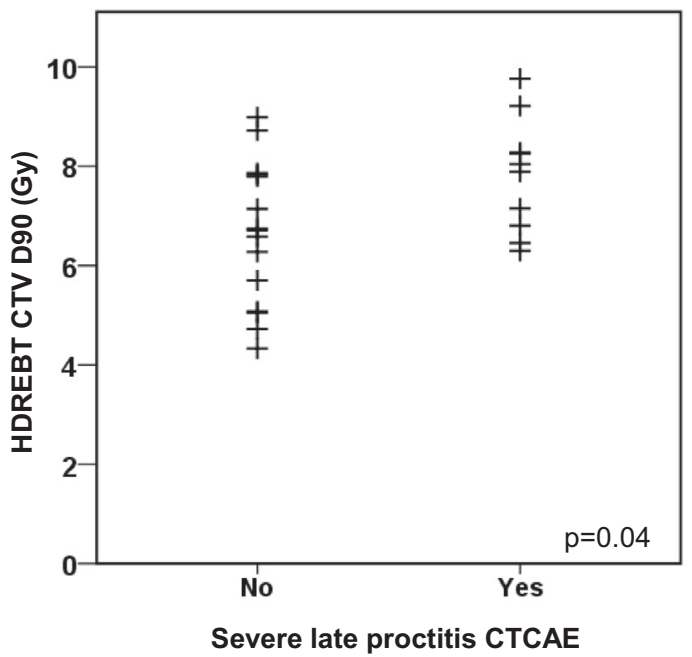

D

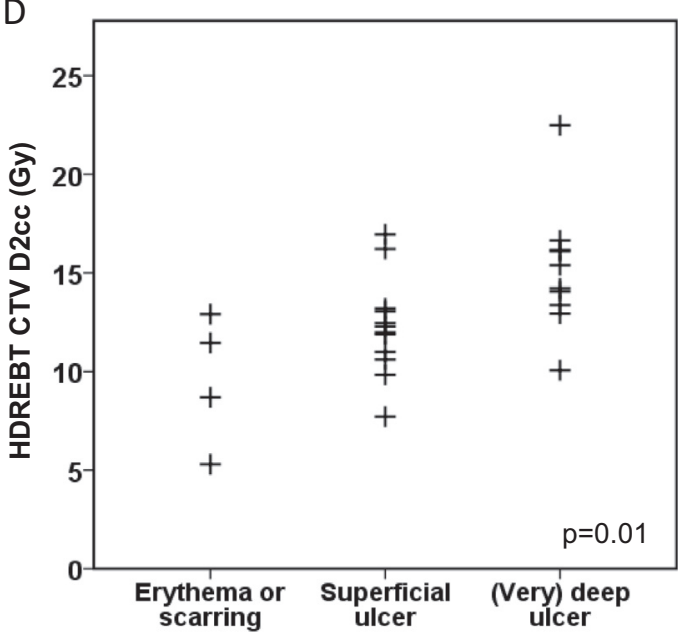

Endoscopic toxicity at tumor site

Fig. 3. Factors associated with rectal toxicity. HDREBT: high-dose rate endorectal brachytherapy, CTV: clinical target volume. A: Correlation of HDREBT CTV D90 (Gy) with acute proctitis CTCAE. B: Correlation of HDREBT CTV D90 (Gy) with severe late proctitis CTCAE. C: Correlation of HDREBT CTV volume (cc) with endoscopic toxicity at the tumor site. D: Correlation of HDREBT CTV D2cc (Gy) with endoscopic toxicity at the tumor site.

In our study, a higher dose to the brachytherapy CTV (D90) was associated with patient and physician reported proctitis and brachytherapy CTV volume and CTV D1cc and D2cc were correlated with ulceration at the tumor site. No association was observed for patient comorbidities or dose to the normal rectal wall. Our study population however existed entirely of elderly/frail patients with comorbidity who were therefore all at increased risk of rectal toxicity. Despite the absence of a clear dose-response correlation, it is advisable to limit the dose in the normal mucosa as much as possible.

Limitations of this study are the small number of patients and multiple tests performed, making it on the one hand difficult to distinguish between real effects and random variations, whereas on the other hand real effects might remain undetected. Also, we included patients with a partial response in the toxicity analyses and have to consider the following in the interpretation of the results. Firstly, proctitis could partly be caused by residual tumor limiting the correlation with other parameters, and secondly, both toxicity and tumor regression can result in ulceration. We observed a correlation for both volume and CTV D2cc with ulceration at the tumor site, which is likely a combined result of regression in large tumors and ulceration after high doses to the mucosa.
The HDREBT technique has evolved since the HERBERT study and the current findings have to be validated in future studies using optimal brachytherapy treatment planning with incorporation of repeated CT-scanning before every fraction [9,24]. The use of spacing balloon(s) to improve applicator positioning, together with optional use of shielding in the central canal, contribute to decreasing dose to organs at risk [24]. Use of MRI with applicator in situ, has the advantage of visualization of the residual GTV at time of brachytherapy, and has been shown to improve the reproducibility of target delineation in other sites [25,26]. MRI would also allow the use of an adaptive target concept that takes tumor regression during treatment into account and can further direct dose optimization for areas at risk of macroscopic residual tumor and at risk for microscopic disease. Consensus on target definition and dose reporting are needed to further improve the understanding of dose-effect relationships for HDREBT from an international perspective.

The findings of the current study can be useful in selection of patients for definitive radiotherapy. It seems that patients with a baseline tumor volume $<20 \mathrm{cc}$ and at least a partial response to EBRT are good candidates for a brachytherapy boost. Use of anticoagulants increases the risk of late rectal blood loss and this should 
be considered when counseling patients for this treatment. As previously reported, the recommended brachytherapy boost dose after 13x3Gy EBRT was determined to be $7 \mathrm{~Gy}$ per fraction [6]. Based on the current analyses we advise to aim for a maximum CTV/rectal wall D2cc of $200 \%$ (14 Gy/\#) to limit the risk of deep ulceration. While some of the patients that were treated with 7 Gy per fraction did experience severe late proctitis, we expect that with improved patient selection, technique and the suggested additional constraint we will observe less severe toxicity. Further research on the added value and risks of a HDREBT boost in elderly patients with limited treatment options is necessary. A proposed follow-up study for medically inoperable rectal cancer patients (HERBERT II) will randomize between EBRT alone and EBRT followed by a HDREBT boost using these treatment planning aims.

\section{Disclaimers}

Nucletron/Elekta contributed to the applicators used in this study.

\section{Conflicts of interest}

None.

\section{Appendix A. Supplementary data}

Supplementary data to this article can be found online at https://doi.org/10.1016/j.radonc.2019.01.034.

\section{References}

[1] Beets GL, Figueiredo NL, Habr-Gama A, van de Velde CJ. A new paradigm for rectal cancer: Organ preservation: Introducing the International Watch \& Wait Database (IWWD). Eur J Surg Oncol 2015;41:1562-4.

[2] Marijnen CA. Organ preservation in rectal cancer: have all questions been answered? Lancet Oncol 2015;16:e13-22.

[3] Smith FM, Rao C, Oliva Perez R, Bujko K, Athanasiou T, Habr-Gama A, et al. Avoiding radical surgery improves early survival in elderly patients with rectal cancer, demonstrating complete clinical response after neoadjuvant therapy: results of a decision-analytic model. Dis Colon Rectum 2015;58:159-71.

[4] Appelt AL, Ploen J, Vogelius IR, Bentzen SM, Jakobsen A. Radiation doseresponse model for locally advanced rectal cancer after preoperative chemoradiation therapy. Int J Radiat Oncol Biol Phys 2013;85:74-80.

[5] Ortholan C, Romestaing P, Chapet O, Gerard JP. Correlation in rectal cancer between clinical tumor response after neoadjuvant radiotherapy and sphincter or organ preservation: 10-year results of the Lyon R 96-02 randomized trial. Int J Radiat Oncol Biol Phys 2012:83:e165-71.

[6] Rijkmans EC, Cats A, Nout RA, van den Bongard D, Ketelaars M, Buijsen J, et al. Endorectal brachytherapy boost after external beam radiation therapy in elderly or medically inoperable patients with rectal cancer: primary outcomes of the phase 1 HERBERT Study. Int J Radiat Oncol Biol Phys 2017:98:908-17.

[7] Buckley H, Wilson C, Ajithkumar T. High-dose-rate brachytherapy in the management of operable rectal cancer: a systematic review. Int J Radiat Oncol Biol Phys 2017:99:111-27.

[8] Rijkmans EC, van Triest B, Nout RA, Kerkhof EM, Buijsen J, Rozema T, et al. Evaluation of clinical and endoscopic toxicity after external beam radiotherapy and endorectal brachytherapy in elderly patients with rectal cancer treated in the HERBERT study. Radiother Oncol 2018:126:417-23.

[9] van den Ende RPJ, Rijkmans EC, Kerkhof EM, Nout RA, Ketelaars M, Laman MS, et al. Benefit of adaptive CT-based treatment planning in high-dose-rate endorectal brachytherapy for rectal cancer. Brachytherapy 2018;17:78-85.

[10] Devic S, Vuong T, Moftah B, Evans M, Podgorsak EB, Poon E, et al. Image-guided high dose rate endorectal brachytherapy. Med Phys 2007;34:4451-8.

[11] Khan AM, Birk JW, Anderson JC, Georgsson M, Park TL, Smith CJ, et al. A prospective randomized placebo-controlled double-blinded pilot study of misoprostol rectal suppositories in the prevention of acute and chronic radiation proctitis symptoms in prostate cancer patients. Am J Gastroenterol 2000;95:1961-6.
[12] Marijnen CA. External beam radiotherapy and high dose rate brachytherapy for medically unfit and elderly patients. Clin Oncol ( $\mathrm{R}$ Coll Radiol) 2007;19:706-10.

[13] Aumock A, Birnbaum EH, Fleshman JW, Fry RD, Gambacorta MA, Kodner IJ, et al. Treatment of rectal adenocarcinoma with endocavitary and external beam radiotherapy: results for 199 patients with localized tumors. Int J Radiat Oncol Biol Phys 2001;51:363-70.

[14] Birnbaum EH, Ogunbiyi OA, Gagliardi G, Fry RD, Myerson RJ, Kodner IJ, et al. Selection criteria for treatment of rectal cancer with combined external and endocavitary radiation. Dis Colon Rectum 1999;42:727-33. discussion 33-5.

[15] Rauch P, Bey P, Peiffert D, Conroy T, Bresler L. Factors affecting local contro and survival after treatment of carcinoma of the rectum by endocavitary radiation: a retrospective study of 97 cases. Int J Radiat Oncol Biol Phys 2001;49:117-24.

16] Gerard JP, Frin AC, Doyen J, Zhou FX, Gal J, Romestaing P, et al. Organ preservation in rectal adenocarcinoma (T1) T2-T3 Nx M0. Historical overview of the Lyon Sud - nice experience using contact X-ray brachytherapy and external beam radiotherapy for 120 patients. Acta Oncol 2015;54:545-51.

[17] Gash KJ, Baser O, Kiran RP. Factors associated with degree of tumour response to neo-adjuvant radiotherapy in rectal cancer and subsequent corresponding outcomes. Eur J Surg Oncol 2017;43:2052-9.

[18] van der Sluis FJ, van Westreenen HL, van Etten B, van Leeuwen BL, de Bock GH. Pretreatment identification of patients likely to have pathologic complete response after neoadjuvant chemoradiotherapy for rectal cancer. Int Colorectal Dis 2018;33:149-57.

[19] Garland ML, Vather R, Bunkley N, Pearse M, Bissett IP. Clinical tumour size and nodal status predict pathologic complete response following neoadjuvant chemoradiotherapy for rectal cancer. Int J Colorectal Dis 2014;29:301-7.

[20] Hall MD, Schultheiss TE, Smith DD, Fakih MG, Wong JY, Chen YJ. Effect of increasing radiation dose on pathologic complete response in rectal cancer patients treated with neoadjuvant chemoradiation therapy. Acta Oncol 2016;55:1392-9.

[21] Wiltshire KL, Ward IG, Swallow C, Oza AM, Cummings B, Pond GR, et al Preoperative radiation with concurrent chemotherapy for resectable rectal cancer: effect of dose escalation on pathologic complete response, loca recurrence-free survival, disease-free survival, and overall survival. Int J Radiat Oncol Biol Phys 2006;64:709-16.

[22] Dubben HH, Thames HD, Beck-Bornholdt HP. Tumor volume: a basic and specific response predictor in radiotherapy. Radiother Oncol 1998;47:167-74

[23] Bentzen SM, Thames HD. Tumor volume and local control probability: clinical data and radiobiological interpretations. Int J Radiat Oncol Biol Phys 1996;36:247-51.

[24] Nout RA, Devic S, Niazi T, Wyse J, Boutros M, Pelsser V, et al. CT-based adaptive high-dose-rate endorectal brachytherapy in the preoperative treatment of locally advanced rectal cancer: Technical and practical aspects. Brachytherapy 2016;15:477-84.

[25] Viswanathan AN, Erickson B, Gaffney DK, Beriwal S, Bhatia SK, Lee Burnett O, et al. Comparison and consensus guidelines for delineation of clinical target volume for CT- and MR-based brachytherapy in locally advanced cervical cancer. Int J Radiat Oncol Biol Phys 2014;90:320-8.

[26] Sander L, Langkilde NC, Holmberg M, Carl J. MRI target delineation may reduce long-term toxicity after prostate radiotherapy. Acta Oncol 2014;53:809-14.

[27] Appelt AL, Ploen J, Harling H, Jensen FS, Jensen LH, Jorgensen JC, et al. Highdose chemoradiotherapy and watchful waiting for distal rectal cancer: a prospective observational study. Lancet Oncol 2015;16:919-27.

[28] Fiorino C, Valdagni R, Rancati T, Sanguineti G. Dose-volume effects for norma tissues in external radiotherapy: pelvis. Radiother Oncol 2009;93:153-67.

[29] Michalski JM, Gay H, Jackson A, Tucker SL, Deasy JO. Radiation dose-volume effects in radiation-induced rectal injury. Int J Radiat Oncol Biol Phys 2010;76: S123-9.

[30] Landoni V, Fiorino C, Cozzarini C, Sanguineti G, Valdagni R, Rancati T. Predicting toxicity in radiotherapy for prostate cancer. Phys Med 2016;32:521-32.

[31] Moulton CR, House MJ, Lye V, Tang CI, Krawiec M, Joseph DJ, et al. Prostate external beam radiotherapy combined with high-dose-rate brachytherapy: dose-volume parameters from deformably-registered plans correlate with late gastrointestinal complications. Radiat Oncol 2016;11:144.

[32] Mazeron R, Fokdal LU, Kirchheiner K, Georg P, Jastaniyah N, Segedin B, et al. Dose-volume effect relationships for late rectal morbidity in patients treated with chemoradiation and MRI-guided adaptive brachytherapy for locally advanced cervical cancer: Results from the prospective multicenter EMBRACE study. Radiother Oncol 2016:120:412-9.

[33] Schaake W, van der Schaaf A, van Dijk LV, Bongaerts AH, van den Bergh AC, Langendijk JA. Normal tissue complication probability (NTCP) models for late rectal bleeding, stool frequency and fecal incontinence after radiotherapy in prostate cancer patients. Radiother Oncol 2016;119:381-7. 